\title{
Corrigendum to "Artificial Neural Network to Predict Varicocele Impact on Male Fertility through Testicular Endocannabinoid Gene Expression Profiles"
}

\author{
Davide Perruzza $\mathbb{D}^{1},{ }^{1}$ Nicola Bernabò $\mathbb{D},{ }^{1}$ Cinzia Rapino $\left(\mathbb{D},{ }^{2}\right.$ Luca Valbonetti, ${ }^{1}$ Ilaria Falanga, ${ }^{1}$ \\ Valentina Russo, ${ }^{1}$ Annunziata Mauro $\left({ }^{10},{ }^{1}\right.$ Paolo Berardinelli, ${ }^{1}$ Liborio Stuppia, ${ }^{3}$ \\ Mauro Maccarrone $\mathbb{D}^{4,5}$ and Barbara Barboni $\mathbb{D D}^{1}$ \\ ${ }^{1}$ Faculty of Biosciences and Technology for Food, Agriculture and Environment, University of Teramo, 64100 Teramo, Italy \\ ${ }^{2}$ Faculty of Veterinary Medicine, Agriculture and Environment, University of Teramo, 64100 Teramo, Italy \\ ${ }^{3}$ Department of Psychological, Health and Territorial Sciences, School of Medicine and Health Sciences, University "G. d'Annunzio" of \\ Chieti and Pescara, 66100 Chieti, Italy \\ ${ }^{4}$ Department of Medicine, Campus Bio-Medico University of Rome, 00128 Rome, Italy \\ ${ }^{5}$ European Center for Brain Research, IRCCS Santa Lucia Foundation, 00164 Rome, Italy
}

Correspondence should be addressed to Davide Perruzza; davideperruzza86@gmail.com

Received 27 April 2020; Accepted 28 April 2020; Published 28 May 2020

Copyright (c) 2020 Davide Perruzza et al. This is an open access article distributed under the Creative Commons Attribution License, which permits unrestricted use, distribution, and reproduction in any medium, provided the original work is properly cited.

In the article titled "Artificial Neural Network to Predict Varicocele Impact on Male Fertility through Testicular Endocannabinoid Gene Expression Profiles" [1], information regarding the support of the Italian Ministry for Instruction, University and Research (MIUR), was omitted in the Acknowledgments section. The corrected section appears below:

\section{Acknowledgments}

We express our gratitude to Dr. Delia Nardinocchi (University of Teramo, Italy) for her technical support in histological analyses and to Dr. Filomena Fezza and Dr. Monica Bari (Tor Vergata University of Rome, Italy), for their kind assistance with the qRT-PCR experiments. This work has been funded by the Project PRIN: "Environment, lifestyle and iatrogenic effects on sperm DNA genetic and epigenetic modifications: consequences for male reproductive fitness" (Nicola Bernabò) and supported by the Italian Ministry for Instruction, University and Research (MIUR) under the National Operative Program (PON): Programma Operativo Nazionale Ricerca e Innovazione 2014-2020 (CCI 2014IT16M2OP0005), Fondo Sociale Eur- opeo (FSE), Azione I.1 "Dottorati Innovativi con caratterizzazione Industriale" (grant number C42F16000360001).

\section{References}

[1] D. Perruzza, N. Bernabò, C. Rapino et al., "Artificial neural network to predict varicocele impact on male fertility through testicular endocannabinoid gene expression profiles," BioMed Research International, vol. 2018, Article ID 3591086, 15 pages, 2018. 